\title{
Comparison of two topical medications, on pain relief due to fistula cannulation in hemodialysis patients
}

\author{
Farideh MALEKSHAHI, Shirzad FALLAHI, Mehdi MOHSENI, Mohammad ALMASIAN
}

\begin{abstract}
Patients with end stage renal failure consider the pain caused by recurrent fistula cannulations as the most intense stress they experience from their treatment and the biggest concern in their lives. One of the fundamental objectives of the nursing procedures is to relieve this pain. Therefore, this study aimed to determine the impact of two topical medications, namely Piroxicam and EMLA cream, on the pain caused by fistula cannulation among hemodialysis patients. This was a clinical trial conducted on 75 patients referring to the dialysis unit of the Shohadaye Ashayer Hospital of Khorramabad, West of Iran, in 2013. The patients were randomly divided into three groups: group A (Piroxicam), group B (EMLA cream), and group C (placebo). The data collection instrument was a questionnaire consisting of three parts: demographic information, the visual analog scale (VAS) for pain assessment, and a checklist for the likely side effects of the medications. The severity of pain during fistula cannulation was assessed in the three groups in two stages
\end{abstract}

(before and after the intervention). To analyze the data, the Kruscal-Wallis and the Mann-Whitney statistical tests and SPSS 19 were used. The median for pain relief was obviously higher in the EMLA cream group than in either the Piroxicam or the placebo group and this difference was statistically significant $(p<0.001)$. Comparing the Piroxicam and the placebo showed that the pain reduction median was much higher in the Piroxicam group than the placebo group, but the difference was not significant. The results of this study showed that the EMLA cream is more effective than the Piroxicam gel in the reduction of pain caused by fistula cannulation among hemodialysis patients. Therefore, based on the results of the present study, it can be suggested that the EMLA cream, as a simple treatment method which can be applied by the patient himself/herself, be used to relieve pain during fistula cannulation of hemodialysis patients.

Keywords: Topical medications; pain relieve; fistula cannulation; hemodialysis patients
Farideh Malekshahi

Department of Health, School of Nursing, Lorestan University of Medical Sciences, Khorramabad, Iran

Shirzad Fallahi

Razi Herbal Medicines Research Center, Lorestan University of Medical Sciences, Khorramabad, Iran

Mehdi Mohseni

Intensive Care Nursing, Lorestan University of Medical Sciences, Khorramabad, Iran

Mohammad Almasian

Department of the English Language, Lorestan University of Medical Sciences, Khorramabad, Iran

Corresponding Author:

Shirzad Fallahi

e-mail:Shfupdate@gmail.com, Falahi.sh@lums.ac.ir

Submitted / Gönderilme: 16.03.2017 Revised / Düzeltme: 05.06.2017 Accepted / Kabul: $\quad 06.06 .2017$

How to cite this article : Malekshahi F, Fallahi S, Mohseni M, Almasian M. Comparison of two topical medications, on relieve the pain due to fistula cannulationinhemodialysispatients.MarmaraPharmJ2017;21(4):1002-1009

\section{Introduction}

Pain is an unpleasant feeling and an emotional experience which accompanies potential or actual tissue damage [1]. Pain not only results from major disease, but it may also derive from invasive diagnostic and treatment procedures [2]. Diagnostic and treatment procedures have been recognized as the most stressful medical events [3]. Studies have shown that $5.3 \%$ of adult patients suffer from an intense fear of needles and $22 \%$ suffer from moderate fear. The pain resulting from the insertion of needles and catheters into blood vessels is problematic when it is recurrent and it is necessary to use them continuously. For instance, in the treatment of patients suffering from chronic kidney failure, hemodialysis as a maintenance therapy requires the continuous use of arterial and venous needles for the patient [4].

Given the fact that these patients usually undergo two fistula cannulations 3 times a week, they experience the pain of the 
needle at least 300 times each year $[5,6]$. Studies show that $47 \%$ of hemodialysis patients are afraid of needles, and report the insertion of vascular catheters as the most intense stress resulting from treatment and their biggest concern at the time of hemodialysis [7], and more than one fifth of hemodialysis patients consider the pain caused by the insertion of vascular needles unbearable [4]. Annually, millions of people suffer from this disease and a lot of money is spent for the treatment of this disease in the health care system. The prevalence of kidney failure involves 3500 new cases each year, and it is predicted that a prevalence of more than 1000 cases per each million people is likely in the developing countries [8]. In Iran, the average growth rates for this disease are higher than the mean global growth rates, being $12 \%$ per year. Based on the reports of the Iranian Research Center for Kidney Diseases and Kidney Transplants, in 2007, about 29000 patients suffering from end stage kidney failure were receiving treatment, from among whom 14000 patients (48.5\%) were treated using hemodialysis [8]. Based on available reports, the prevalence of this disease is increasing worldwide $[10,11]$.

Among health care staff, nurses play a key role in pain relief. During the previous decades, the role of nurses in pain relief has received increasing attention, and various studies have shown that the role and importance of the nursing staff in pain control is expanding. Due to the pivotal role they play in the treatment and care of patients suffering from chronic kidney failure, nurses can be effective in relieving and reducing the pain these patients experience [12]. To achieve this goal, it is necessary to use simple, efficacious, and cost-effective methods to reduce and relieve pain and to try to prevent their negative side effects [13]. There are many medicinal and non-medicinal methods available to relieve pain. Topical anesthesia is one of the common methods that can be used to overcome the pain resulting from cannulation [14]. Some of its advantages include localized action of this method with minor systemic absorption, easy prescription, and its applicability by the patients themselves [15]. One topical anesthetic is the EMLA CREAM, which is a eutectic mixture consisting of lidocaine $2.5 \%$ and prilocaine $2.5 \%$ [16], which blocks the conduction of the nerve impulses by changing the depolarization of the cell membrane to the sodium ion [17]. This medicine permeates healthy skin and creates anesthesia in the surface layers of the skin for several millimeters [18]. For maximum effectiveness, the cream must be applied to the target area and the area must be bandaged about 60 minutes before the main treatment $[18,19]$. From among the side effects of the EMLA CREAM, the bleaching of the skin can be mentioned. To prevent skin bleaching which is due to venous constriction [20], researchers recommend that venipuncture should be performed after 10 minutes [21]. To prevent methaemoglobinaemia, which is caused by prilocaine, this medication should not be administered to patients suffering from glucose-6-phosphate dehydrogenase deficiency, those suffering from congenital or idiopathic methaemoglobinaemia, infants younger than 12 months old, and individuals receiving sulfonamides, nitrate, or phenobarbital [22].

Piroxicam is another topical analgesic medication, which is also a non-steroidal anti-inflammatory drug. The topical form of this drug has been used to relieve the pains of skeletal muscles, to induce analgesia, to reduce the pain and inflammation resulting from venous cannulation, to reduce the pain and inflammation caused by laser removal of unwanted hair, and to reduce the periodical pain in the breasts [23-25]. The topical Piroxicam gel reduces pain and inflammation by preventing the synthesis of prostaglandins and affecting peripheral nociceptors [26]. Patients lacking kidney function can continue to live for many years with the help of hemodialysis. These patients have to cope with not only the stress of suffering from this disease, but they also have to deal with the stress and pain of repeated cannulations, all of which compromise the quality of life of these patients. Given the fact that one of the duties of nurses is to relieve or minimize the pain the patients experience, nurses should take any available measures to reduce the pain experienced by hemodialysis patients during the insertion of vascular needles. Based on the importance of the issue and given the fact that, up to now, no studies have been conducted in Iran regarding the application of topical medications to reduce the pain resulting from the fistula cannulation of dialysis patients, this study aimed to determine the effects of the two topical medications of Piroxicam and EMLA CREAM on the pain intensity experienced by hemodialysis patients as a result of fistula cannulation.

\section{Materials and Methods}

\section{Study population and design}

This was a double-blind clinical trial conducted on 75 patients referring to the dialysis unit of the Shohadaye Ashayer Hospital of Khorramabad, West of Iran, in 2013. The patients were randomly assigned to three groups (A or Piroxicam, B or EMLA cream, and $\mathrm{C}$ or placebo (vitamin $\mathrm{A}+\mathrm{D})$ ) using the stratified block randomization method, such that the patients were stratifies in terms of age group, sex, educational attainment level, and the length of time they had undergone 
fistula cannulations, and in each stratum, they were assigned to three groups based on a table of random numbers and blocks consisting of six participants. The inclusion criteria included being older than 18 years old, having a history of at least three months of hemodialysis, not being verbally, mentally, or visually disabled, not having a history of psychiatric diseases and hospitalization in psychiatric wards, not being addicted to analgesics, feeling no pain before cannulation, not having taken a painkiller during the past 24 hours, not having a wound in the fistula cannulation site, not having a history of favism or congenital or idiopathic methemoglobinemia, not suffering from hepatic diseases, asthma, skin allergies, dermatitis, and active digestive tract bleeding, and not being treated with sulfonamides, nitrate, or phenobarbital. The consent of the patients for participation in the study and the necessary permissions from the university were obtained. The patients were able to leave the study at any time they wished. The exclusion criteria included not giving consent and not meeting the study criteria.

\section{Data collection instrument}

The data collection instrument consisted of demographic information, a visual scale for measuring the intensity of pain, and a checklist to assess the short-term side effects of the medications. The demographic information included age, sex, educational attainment level, and the length of time they had used fistula cannulations. The visual scale used for the measurement of pain intensity was a $10 \mathrm{~cm}$ ruler, on the leftmost end of which the words "no pain" and on the right-most end of which the words "the most intense pain" were written. After fistula cannulation, the patients were asked to indicate the amount of pain on a scale of 0 to 10 . The visual scale of the measurement of pain intensity is a standard instrument and has been widely used in Iran. The scientific reliability and validity of the visual pain measurement scale have been investigated in numerous studies. Regarding this instrument, the results of the study by Williamson and Hoggart (2005) showed that this instrument has appropriate reliability and validity and can be used in treatment [27]. Furthermore, nursing reference books have pointed to the reliability and validity of this instrument [28].

\section{Intervention and Follow-up Measurements}

The checklist for assessing the likely short-term side effects of the medications included items related to bleaching, erythema, edema, and hardening of the skin which were assessed and recorded after the removal of the bandage and the sterilization of the skin and before the cannulation. In the first session, after the routine fistula cannulation, the patients were asked to indicate the amount of pain on the visual scale to measure the pain and the obtained data were recorded. At the end of the first session of dialysis, the researcher put at the disposal of each patient one tube of the medicine (given the group the patient belonged to), the outer surface of which was covered with adhesive tapes, together with a transparent wound dressing (bandage). The patients were instructed to apply 2 grams of the medicine to the place of insertion of the needle in the fistula and cover the area with the transparent bandage before the next session of dialysis (the second session) one hour before referring to the dialysis unit. In the second session, before cannulation, the bandage was removed, the area was sterilized by alcohol, and to prevent the venous constriction caused by the medication (the EMLA cream), 10 minutes after the sterilization, the cannulation was performed in the three groups. All cannulations were performed with the same size needle (hemodialysis needle number 16) by skilled nurses working in the hemodialysis unit and in case the first attempt at cannulation was unsuccessful and a reinsertion was needed, the participant was excluded from the study. To analyze the data, the Kruskal-Wallis and the Mann-Whitney tests and the SPSS application version 19 were used with a significance level of 0.05 .

\section{Ethical approval}

Ethical considerations were taken into account in this study (Ethical approval number; 215/144-2013). Sampling was conducted after obtaining permissions from the Ethics Committee of the Lorestan University of Medical Sciences, the people in charge of the hospital, and the dialysis unit. After introducing himself/herself to the participants, the researcher would adequately explain the objectives of the study, the procedures, the voluntary nature of the participation, and the confidentiality of the data, and written consents were obtained.

\section{Results}

\section{Demographic variables}

The findings of the study regarding the demographic variables such as age, sex, educational attainment levels, and the length of time the patients had used fistula cannulations indicated that the groups were similar in terms of these variables and showed no significant differences. Based on the findings, $64 \%$ of the patients in each group were male 
and $36 \%$ were female. Based on the obtained results, in each group, $4 \%$ of the patients were younger than $30,24 \%$ were between 30 to 49 years old, and $72 \%$ were older than 50 years old. The results regarding the age of the fistula showed that 9.3\% of the patients had used a fistula for less than a year, $34.7 \%$ had used fistulae between 1 year and 3 years, and $56 \%$ of the patients had used fistulae for more than 3 years. The individual characteristics of the studied patients are presented in Table 1.

\section{Intervention and Follow-up Measurements}

Regarding the objectives of the study, the results showed that there was a statistically significant difference between the median pain reduction before and after the intervention (given the study group) $(P<0.001)$. Additionally, the findings indicate that there are significant differences among the median of the pain intensity as experienced by the three groups. Comparing the groups two by two showed that the median pain reduction in the EMLA cream group was obviously higher than the Piroxicam and the placebo groups and this difference was significant $(P<0.001)$. The comparison between the Piroxicam and the placebo group showed that the median pain reduction was much higher in the Piroxicam group than in the placebo group, but this difference was not significant (Table 2).

Regarding the results obtained about the possible shortterm side-effects of the medications (Piroxicam, EMLA cream, and vitamin A $+\mathrm{D})$, including bleaching, erythema,

Table 1. The demographic characteristics of the studied hemodialysis patients

\begin{tabular}{|c|c|c|c|}
\hline \multicolumn{2}{|c|}{ Demographic characteristics } & \multirow{2}{*}{$\begin{array}{c}\text { Number } \\
3 \\
\end{array}$} & \multirow{2}{*}{$\begin{array}{c}\text { Percentage } \\
4\end{array}$} \\
\hline Age (years) & Younger than 30 & & \\
\hline & $30-49$ & 18 & 24 \\
\hline & Older than 50 & 54 & 72 \\
\hline \multirow[t]{2}{*}{ Gender } & Female & 27 & 36 \\
\hline & Male & 48 & 64 \\
\hline \multirow[t]{4}{*}{ Education } & Illiterate & 61 & 81.3 \\
\hline & Primary school-Secondary school & 6 & 6.7 \\
\hline & High school-High school diploma & 6 & 8 \\
\hline & University & 3 & 4 \\
\hline \multirow[t]{3}{*}{ Fistula age } & Less than 1 year & 7 & 9.3 \\
\hline & $1-3$ years & 26 & 34.7 \\
\hline & 3 years and more & 42 & 56 \\
\hline
\end{tabular}

Table 2. Comparing the amount of pain experienced by the patients before and after the intervention separately in each groups

\begin{tabular}{|c|c|c|c|c|c|c|c|c|c|}
\hline \multirow{3}{*}{ Criteria } & \multicolumn{9}{|c|}{ Group } \\
\hline & \multicolumn{3}{|c|}{$\begin{array}{c}\text { Piroxicam } \\
(\text { Number }=25) \\
(\text { Male }=16, \text { Female }=9)\end{array}$} & \multicolumn{3}{|c|}{$\begin{array}{c}\text { EMLA } \\
(\text { Number }=25) \\
(\text { Male }=16, \text { Female }=9)\end{array}$} & \multicolumn{3}{|c|}{$\begin{array}{c}\text { Placebo } \\
(\text { Number }=25) \\
(\text { Male }=16, \text { Female }=9)\end{array}$} \\
\hline & Before & After & Reduction & Before & After & Reduction & Before & After & Reduction \\
\hline Mean & 4.28 & 2.88 & 1.40 & 3.84 & 1.36 & 2.48 & 3.92 & 3.16 & 0.76 \\
\hline Median & 5 & 2 & 1 & 4 & 1 & 3 & 4 & 3 & 0 \\
\hline Standard deviation & 1.93 & 2.39 & 2.18 & 1.65 & 1.22 & 1.58 & 2.14 & 2.25 & 1.71 \\
\hline Interquartile range & 2.5 & 4 & 2 & 2.5 & 1.5 & 2 & 2.5 & 3.5 & 1 \\
\hline Pvalue & & & & & & $0.001^{*}$ & & & \\
\hline
\end{tabular}


edema, and hardening, one hour after the application of the medications in the fistula site and before cannulation, it can be mentioned that the highest percentage of the short-term side effects belonged to bleaching in the EMLA CREAM group (16\%), and in the remaining $84 \%$ of the participants of this group, and $100 \%$ of the participants of the other two groups (Piroxicam and vitamin A + D) no possible shortterm side-effects were observed.

\section{Discussion}

Although the number of specialized studies conducted on the pain caused by fistula cannulation of hemodialysis patients is limited, many studies have been conducted to determine the efficacy of medicinal and non-medicinal methods on pain intensity among patients at the time of insertion of the needle into the vessels. The present study, too, has been conducted to determine the effects of Piroxicam and EMLA CREAM on the intensity of the pain caused by fistula cannulation among dialysis patients. Based on the findings of the present study, the majority of the patients were older than 50. In the study by Asgari et al. (2013), the mean age of the studied patients was 52.46 [29]. Vosughi (2009) reported the mean age of the studied patients as 55.05 [30]. Given the facts that the most common causes of end stage kidney failure are diabetes and hypertension and that the incidence of these diseases increases with age [31], therefore most patients suffering from chronic kidney failure are among older adults.

The results of the present study regarding gender showed that the majority of the patients in each group were male. In a study by Van Loon et al. on the complications and pain of two technique of insertion of the hemodialysis needles conducted on 145 patients, the percentage of male participants was $63 \%$ and the percentage of female patients was $37 \%$ [32]. Additionally, in the study by Asgari et al. (2013) $70 \%$ of the patients were male and $30 \%$ were female [29]. As can be observed, in related studies as in the present study, the number of male hemodialysis patients is higher than the number of female patients. This can be due to various causes. Monfared et al. (2003), in a study that examined the causes of chronic kidney failure among the hemodialysis patients of the Gilan Province of Iran, reported hypertension as the most common factor contributing to kidney failure among hemodialysis patients [33]. A study by Noblat et al. (2004) showed that the prevalence of kidney failure consequent to hypertension was higher among males than females hospitalized in intensive care units [34]. Therefore, given the factors contributing to the occurrence of chronic kidney failure and the studies conducted on this issue, the higher number of male hemodialysis patients in this study is justifiable.

Regarding the objectives of the present study, i.e. determining pain intensity during fistula cannulation in dialysis patients, it can be said that there was a statistically significant difference between the median pain reduction before and after the intervention (regardless of which group was considered), meaning that the intervention was effective in reducing pain in the three groups. Moreover, the findings indicate that the difference among the median pain intensities in the three groups was also significant. A two-by-two comparison of the groups showed that the median pain intensity reduction was higher in the EMLA cream group than in the Piroxicam and the placebo groups, and this difference was significant. Comparing the Piroxicam and the placebo groups also showed that the median pain reduction in the Piroxicam group was considerably higher than the placebo group, but this difference was not significant. About comparing the effects of the EMLA cream and the Piroxicam gel with the placebo, Dutta et al. (2003) in a clinical trial concluded that the EMLA cream significantly reduced the pain of venipuncture more than the Piroxicam gel [23]. Agarwal et al. (2007) reported a $63 \%$ reduction in the pain caused by venipuncture in the EMLA CREAM group as compared with the placebo group (Vaseline patch) and a 52\% pain reduction in the diclofenac patch group compared with the placebo group [35]. In a clinical trial aiming to compare the application of EMLA spray and placebo on the reduction of pain resulting from cannulation, Celik et al. (2011) concluded that EMLA was effective in comparison with placebo in reducing pain $(P<0.05)$ [36]. The results of a controlled clinical trial by Ingalls et al. (2010) on the application of lidocaine patches $5 \%$ in reducing the pain resulting from rib fracture demonstrated that no significant difference existed between the experimental group and the control group [37].

Moore et al. (1998) in a review study concluded that topical non-steroidal anti-inflammatory drugs including Piroxicam are significantly more effective than placebo in treating chronic pains [38]. Furthermore, a study by Khalili et al. (2012) demonstrated that the pain caused by venipuncture can be significantly lower when the EMLA cream is applied than when the Vaseline ointment is used [39]. Many studies have compared various medications in different forms and combinations with the EMLA cream in terms of controlling the feeling of pain during painful medical procedures. In line with the present study, Galinkin et al. (2002) compared lidocaine iontophoresis with the EMLA cream in reducing 
the pain caused by venipuncture in children and concluded that both methods equally reduce pain, however, lidocaine iontophoresis cannot be administered to everyone due to the use of an electric current [40]. On the other hand, Asgari et al. (2013) conducted a clinical trial to investigate the effect of lidocaine spray on pain intensity during the insertion of vascular needles in hemodialysis patients and found out that the mean pain intensity was significantly different between the lidocaine spray group and the placebo group [29].

Consistent with the present study, Celik et al. (2011) showed that the EMLA cream significantly reduces the cannulation pain the hemodialysis patients experience more than Vapocoolant and placebo [36]. Other studies, e.g. Pourmovahed et al. (2008) [41] and Nikfarid et al. (2010) have confirmed the results of our study regarding the effectiveness of the EMLA cream on the reduction of pain [42]. Additionally, Agarwal et al. (2007) found out that the highest percentage of side effects (during the first hour) in the EMLA group belonged to skin bleaching and, in the diclofenac patch group, no side effects were observed, which is in line with the results of the present study [35]. Regarding the side-effects of the medications, the results showed that skin bleaching occurred among only some EMLA CREAM group patients, and the other members of the EMLA cream group, the Piroxicam group, and the placebo group experienced no side-effects. The results of the present study are consistent with the results of other studies. In a study by Khalili et al. (2012), in $20 \%$ of the participants of the EMLA cream group, skin bleaching was observed, while no side-effects were observed in the placebo group [39]. Moreover, in the study by Dutta et al. (2003), the highest percentage of short-term sideeffects (skin bleaching) was observed in the EMLA CREAM group, while no side-effects were observed in the Piroxicam group [23]. Additionally, in the study by Pérez-Pérez et al. (2006), the application of EMLA resulted in positive allergies, which disappeared after treatment with topical corticosteroids [43]. Moreover, in the study by Wehle et al. (1989), in a doubleblind study on the application of EMLA in the reduction of pain in hemodialysis patients, the results showed that there was a significant decrease in pain in the patients. However, no side effects were observed, except for two patients who suffered topical irritation [44]. It has been mentioned that the reason for the skin bleaching due to the application of the EMLA cream is the effect of this medication on the vessels (vascular constriction) and it has also been said that this side-effect is temporary and relieves by itself [20]. Regarding Piroxicam, in addition to showing no side-effects during the first hour after application, some studies have shown that this medication can prevent and even treat phlebitis consequent to venipuncture.
In this regard, Haji Hosseini et al. (2007) found out that Piroxicam can significantly reduce the likelihood of phlebitis as compared with warm compress and the control group [45]. Bergquist et al. demonstrated that Piroxicam can significantly treat surface thrombophlebitis [46]. Based on these studies, Piroxicam gel can both control the acute pain caused by cannulation and prevent the occurrence of a set of symptoms including pain, inflammation, reddening, induration, and swelling subsequent to the cannulation process.

\section{Conclusion}

Given the findings, it can be concluded that the EMLA cream is more effective than Piroxicam gel in relieving the pain caused by fistula cannulation in hemodialysis patients. Therefore, based on the results of the present study, it can be suggested that the EMLA cream, as an easy method that can be applied by the patients themselves, be used to reduce the pain experienced during fistula cannulation among hemodialysis patients.

\section{Declaration of Interest}

The authors declare that there is no conflict of interest in this study.

\section{Acknowledgments}

The researchers would like to extend their heartfelt thanks to the officials of the Lorestan University of Medical Sciences who allowed and supported this study. This student thesis has been registered at IRCT with the following registration number. IRCT $=2013051213301 \mathrm{~N} 1$. They would also like to express their gratitude to the staff of the dialysis unit of the Shohadaye Ashayer Hospital of Khorramabad, West of Iran, who cooperated with the researchers during data collection. The researchers also would also like to thank all hemodialysis patients who participated in this study.

\section{Limitations of research}

Among the limitations of the study, the exact time and amount at which the creams were applied (one hour before referral, 2 grams) which were not easily controllable. However, the necessary explanations and training were offered and the patients were reminded of the instructions to reduce error. Moreover, another limitation of the study was uncooperativeness of some nurses in timely cannulation. 


\section{References}

1. Schere JC, Timby BK, Smith NE. Introductory Medical Surgical Nursing. Lippincott Co, W.B. Philadelphia. 1999, pp 346.

2. Boggs Underman K, Arnold E. Interpersonal Relationships: professional communication skills for nurses, Fourth Ed. Saunders Company. 2003, pp 346.

3. Brenna A. Caring for children during procedure: A review of the literature. Pediatric Nurs 1994; 20: 457.

4. Harris TJ, Nazir R, Khetpal P, Peterson RA, Chava P, Samir SS, Kimmel PL. Pain, sleep disturbance and survival in hemodialysis patients. Nephrol Dial Transplant 2012; 27: 75865.

5. P B S, Khakha DC, Mahajan S, Gupta S, Agarwal M, Yadav SL. Effect of cryo-therapy on arteriovenous fistula puncturerelated pain in hemodialysis patients. Indian J Nephrol 2008; 18:155-8.

6. Quinn RR, Lamping DL, Lok CE, Meyer RA, Hiller JA, Lee J, Richardson EP, Kiss A, Oliver MJ. The vascular access questionnaire: Assessing patient-reported views of vascular access. J Vasc Access 2008; 9:122-8.

7. McLaughlin K, Manns B, Mortis G, Hons R, Taub K. Why patients with ESRD do not select self-care dialysis as a treatment option. Am J Kidney Dis 2003; 41: 380-5.

8. Zakeri Moghadam M, Aliasgharpoor M. Comprehensive Book. Intensive nursing cares in CCU, ICU, and Dialysis wards; (In Persian). Andishe Rafie. 2009, pp 277-362.

9. Aghighi M, Rouchi HA, Zamyadi M, Mahdavi-Mazdeh M, Norouzi SH, Rajolani H, Ahrabi S, Zamani M. Dialysis in Iran. Iranian J Kidney 2008; 2: 11-5.

10. Burk K, Lemone P. Medical-surgical nursing care, 2nd edition. Pearson Education, New Jersey. 2007, pp 234.

11. Malek F, Tousi J, Tamadon M, Mousavy Sh, Malek M, Ghaderi M. The roles of filters in hypoxia at hemodialysis patients. J. Shahid Sadoughi Univ Med Sci 2008; 16: 34-9. (In Persian)

12. Tanyi RA, Werner JS. Women's Experience of spirituality within end-stage renal disease and hemodialysis. Clin Nurse Res 2008; 17: 32-49.

13. Reis EC, Roth EK, Syphan JL, Tarbell SE, Holubkov R. Effective pain reduction for multiple immunization injections in young infants. Arch Pediatrics Adolescent Med 2003; 157:1115-20.

14. Irsfeld S, Klement W, Lipfert P. Dermal anesthesia: comparison of EMLA CREAM with iontophoretic local anaesthesia. British J Anesthesiol 1993; 71: 375-8.

15. Minassian VA, Jazayeri A, Prien SD, Timmons RL, Stumbo K. Randomized trial of lidocaine ointment versus placebo for the treatment of postpartum perineal pain. Obstetric Gynecol 2002; 100: 1239-43.

16. Manual of dermatologic theraqeutic. Editor : Ardent K. Little Brown, USA. 1995, pp 261-262.

17. Tanyal R, Ostrow $\mathrm{Cl}$. The use of EMLA CREAM to decrease venipuncture pain in children. Pediatric Nurs 2004; 202.

18. Wong L. Whaley \& Wong's nursing care of infant and children, 8th edition. Mosby Inc. 2007, pp 205.

19. Buckley MMP. Benfield Eutectic lidocaine/prilocaine cream. A review of the topical anaesthetic/analgesic efficacy of a eutectic mixture of local anaesthetics EMLA CREAM. Drugs 1993; 46: 126-51.

20. De Jong PC, Verburg MP, Lillieborg S. EMLA CREAM ${ }^{\circledR}$ versus ethyl-chloride spray: a comparison of the analgesic efficacy in children. Eur J Anesthesiol 1990; 7: 473-81.

21. Lander JA, Weltman BJ, So SS. EMLA CREAM and amethocaine for reduction of children's pain associated with needle insertion. Cochrane Database System Rev 2006; 3: 004236.

22. Hahn IH, Hoffman RS, Nelson LS. EMLA CREAM-induced methemoglobinemia and systemic topical anesthetic toxicity. J Emerg Med 2004; 26: 85-8.

23. Dutta A, Puri GD, Wig J. Piroxicam gel, compared to EMLA CREAM is associated with less pain after venous cannulation in volunteers. Canadian J Anesthesiol 2003; 50: 775-8.

24. Poelman MC, Piot B, Guyon F, Deroni M, Leveque JL. Assessment of topical non-steroidal anti-inflammatory drugs. J Pharm Pharmacol 1989; 41: 720-2.

25. Ahmadinejad M, Delfan B, Haghdani S, Hashemi M, Khan IA, Tafti MT. Comparing the effect of diclofenac gel and piroxicam gel on mastalgia. Breast J 2010; 16: 213-4.

26. Potter PA, Perry AG. Fundamental of nursing, 6th edition. Mosby, St Louis. 2003, pp 708-711.

27. Williamson A, Hoggart B. Pain: A Review of Three Commonly Used Pain Rating Scales. J Clin Nurs 2005; 14: 798-804.

28. Potter PA, Perry AG. Fundamentals of Nursing, (Translation for Salemi S, Najafi T). Salemi Publication. 2013, pp 502-542.

29. Asgari M, Hoshmand Motlagh N, Soleimani M, Ghorbani R. The effect of lidocaine spray on pain during vascular inserting needles in hemodialysis patients. Komesh 2013; 3: 271-80. (In Persian)

30. Namadi M, Movahedpour A. Quality of life in patients after renal transplantation in comparison with intermittent hemodialysis. J Ardabil Univ Med Sci 2009; 9:171-9. (In Persian)

31. Asgari MR, Soleimani M. Comprehensive book Intensive nursing cares in CCU, ICU, and Dialysis wards, 18th edition. Boshra 2012; 391-3. (In Persian)

32. Van Loon MM, Goovaerts T, Kessels AG, van der Sande FM, Tordoir JH. Buttonhole needling of hemodialysis arteriovenous fistulae results in less complications and interventions compared to the rope-ladder technique. Nephrol Dial Transplantat 2010; 25: 225-30.

33. Monfared A, Orang poor R, Moosavian Roshan Zamir SA, Aghajani Nargesi D. Reasons of chronic renal failure in hemodialysis patients in Guilan province. J. Guilan Univ Medical Sci 2003; 12:76-83. (In Persian)

34. Noblat AC, Lopes MB, Lopes GB, Lopes AA. Complications of hypertension in men and women seen in a referral outpatient care unit. Arq Bras Cardiol 2004; 83: 314-9.

35. Agarwal A, Gautam S, Gupta D, Singh U. Transdermal diclofenac patch vs eutectic mixture of local anesthetics for venous cannulation pain. Canadian J Anesthesiol 2007; 54:196-200.

36. Çelik G, Özbek O, Yılmaz M, Duman I, Özbek S, Apiliogullari S. Vapocoolant spray vs Lidocaine/Prilocaine cream for reducing the pain of venipuncture in hemodialysis patients: 
A randomized, placebo-controlled, crossover study. Int J Med Sci 2011; 8: 623-7.

37. Ingalls NK, Horton ZA, Bettendorf M, Frye I, Rodriguez C. Randomized, double-blind, placebo-controlled trial using lidocaine patch 5\% in traumatic rib fractures . J Am Coll Surg 2010; 210: 205-9.

38. Moore RA, Tramer MR, Carroll D, Wiffen PJ, McQuay HJ. Quantitative systemic review of topically applied nonsteroid anti-inflammatory drugs. BMJ 1998; 316: 333-8.

39. Khalili Shomia S, Safavi M, Yahyavi SH, Farahani H. Assessment of the effect of EMLA cream on vein puncture pain severity with vein catheter in the patients undergoing cesarean section: Randomized placebo controlled trial. J. Mazandaran Univ Sci 2012; 22: 82-8. (In Persian)

40. Galinkin J, Rose J, Harris K, Watcha M. Lidocaine iontophoresis versus eutectic mixture of local anesthetics (EMLA CREAM ${ }^{\circledR}$ ) for IV placement in children. Anest Anal 2002; 94: 1484-8.

41. Pourmovahed Z, Dehghani Kh, Yassinie M, Shakiba M, Tavangar H, Shahrie T. Comparative study of the effect of music distraction and Emla cream on pain of the children during intravenous cannulation. Iran J Nurs 2008; 21: 47-53.
42. Nikfarid L, Ghamar Yousefi R, Namaziyan M, Namdar F, Azam Nezami M. Comparison of EMLA cream versus local refrigeration for reducing venipuncture-related pain in pediatric patients of Children's Medical Center, 2008. J Nursing Res 2010; 5: 32-7.

43. Pérez-Pérez LC, Fernández-Redondo V, Ginarte-Val M, Paredes-Suárez C, Toribio J. Allergic Contact dermatitis from EMLA cream in a hemodialyzed patient. Dermatitis 2006; 17: 85-7.

44. Wehle B, Björnström M, Cedgård M, Danielsson K, Ekernäs A, Gutierrez A, Petterson U, Lindholm T. Repeated application of EMLA cream 5\% for the alleviation of cannulation pain in haemodialysis. Scand J Urol Nephrol 1989; 23: 299-302.

45. Haji Hosseini F, Beheshti Z, Nazari R, Rezai R, Hagi Ahmadi M. Comparison of moist warm compresses and topical gel piroxicam on phlebitis caused by peripheral venous catheter. J Babol Univ Sci. 2006; 9: 33-7. (In Persian)

46. Bergquist D, Brunkwall J, Jensen N, Persson NH. Treatment of superficial thrombophlebitis: A comparative trial between placebo, hirudoid cream and piroxicam gel. Ann Chir Gynaecol 1990; 79: 92-6. 